FACTA UNIVERSITATIS (NIŠ)

Ser. Math. Inform. Vol. 34, No 3 (2019), 511-523

https://doi.org/10.22190/FUMI1903511A

\title{
THE NEW WEIGHTED INVERSE RAYLEIGH DISTRIBUTION AND ITS APPLICATION
}

\author{
Demet Aydin
}

(C) 2019 by University of Niš, Serbia | Creative Commons Licence: CC BY-NC-ND

\begin{abstract}
In this study, a new weighted version of the inverse Rayleigh distribution based on two different weight functions is introduced. Some statistical and reliability properties of the introduced distribution including the moments, moment generating function, entropy measures (i.e., Shannon and Rényi) and survival and hazard rate functions are derived. The maximum likelihood estimators of the unknown parameters cannot be obtained in explicit forms. So, a numerical method has been required to compute maximum likelihood estimates. Finally, the daily mean wind speed data set has been analysed to show the usability of the new weighted inverse Rayleigh distribution.

Keywords: New weighted inverse Rayleigh distribution; Shannon entropy; hazard rate function; Fisher information matrix; wind speed data.
\end{abstract}

\section{Introduction}

The accuracy of procedures in the statistical analysis depends on the suitableness of a distribution used in modeling a data set. Therefore, many statistical distributions have been proposed in the literature because it is very important to determine the distribution which provides the best fit to a data set.

One of the widely-used statistical distributions in the context of reliability studies is the inverse Rayleigh (IR) distribution introduced by Trayer [24]. Sherina and Oluyede [25] stated that the distribution of lifetimes of several types of experimental units can be modeled by the $I R$ distribution. Various extensions of this distribution have been proposed in the literature: transmuted $I R$ distribution [1], modified $I R$ distribution [10], kumaraswamy $I R$ distribution [21] and beta $I R$ distribution [12].

On the other hand, the theory of weighted distributions introduced by Rao [17] and Fisher [3] provides a unifying approach to deal with the problems of model

Received January 09, 2019; accepted March 01, 2019

2010 Mathematics Subject Classification. Primary 60E05; Secondary 62E10 
specification and data interpretation (see [9]). There are more studies on weighted distributions and their applications in various fields including ecology and reliability (see [6], [7], [16], [14], [15], [19], [13] and [4] among the others). Fatima and Ahmad [8] also introduced a weighted IR (WIR) distribution with a single weight function $w(x)=x^{k}$ where $k \geq 0$, and they studied several of its properties.

The objective of the paper is to introduce a new weighted version of $I R$ distribution obtained by using two different weight functions and to discuss its basic characteristics.

The rest of the paper is organized as follows. The new WIR (NWIR) distribution is introduced in Section 2. Some of its statistical and reliability properties are given in Section 3. Equations of maximum likelihood estimates of parameters and a Fisher information matrix are obtained in Section 4. In Section 5, an application of the distribution to real data is presented. Finally, the paper ends with a conclusion.

\section{The New Weighted Inverse Rayleigh Distribution}

Suppose that $X$ is a non-negative random variable with its probability density function $(p d f)$, and $w(x)$ is weight function where $E(w(x))<\infty$. The $p d f$ of weighted distribution of $X$ can be defined as

$$
f_{w}(x)=\frac{w(x) f(x)}{E(w(x))} .
$$

It should be noted that a general class of weight functions $w(x)$ can be defined by

$$
w(x)=x^{i} e^{j x} F^{k}(x)(1-F(x))^{l},
$$

see [23]. Weight functions can be determined for a different combination of $i, j$, $k$ and $l$ values. If we take $w(x)=x^{i}$, then the obtained distribution is called size-biased distribution, and it is length-biased distribution for $i=1$.

Let $X$ be a random variable with the $I R$ distribution having the scale parameter $\lambda$. The $p d f$ and cumulative density function $(c d f)$ of the $I R$ distribution are given by

$$
\begin{aligned}
f(x) & =2 \lambda x^{-3} e^{-\lambda x^{-2}}, x>0, \lambda>0, \\
F(x) & =e^{-\lambda x^{-2}}, x>0, \lambda>0,
\end{aligned}
$$

respectively. Now, substituting the multiplication of weighted functions, $w_{1}(x)=$ $x^{-\alpha}$ and $w_{2}(x)=e^{-\alpha x^{-2}}$, and $p d f$ of IR distribution in (2.1), the $p d f$ of the NWIR distribution is defined by

$$
\begin{aligned}
f_{w}(x) & =\frac{w_{1}(x) w_{2}(x) f(x)}{E\left(w_{1}(x) w_{2}(x)\right)} \\
& =\frac{2(\alpha+\lambda)^{\frac{\alpha}{2}+1}}{\Gamma\left(\frac{\alpha}{2}+1\right)} x^{-(\alpha+3)} e^{-(\alpha+\lambda) x^{-2}}, x>0, \lambda>0, \alpha>0
\end{aligned}
$$


where

$$
\begin{aligned}
E\left(w_{1}(x) w_{2}(x)\right) & =\int_{0}^{\infty} 2 \lambda x^{-(\alpha+3)} e^{-(\alpha+\lambda) x^{-2}} d x \\
& =\frac{\lambda \Gamma\left(\frac{\alpha}{2}+1\right)}{(\alpha+\lambda)^{\frac{\alpha}{2}+1}}<\infty .
\end{aligned}
$$

It should be noted that the following transformation is applied in order to calculate $E\left(w_{1}(x) w_{2}(x)\right)$

$$
u=(\alpha+\lambda) x^{-2} \Longrightarrow x=\sqrt{\frac{\alpha+\lambda}{u}} \Longrightarrow d u=-2(\alpha+\lambda) x^{-3} d x .
$$

The corresponding $c d f$ of the NWIR distribution is

$$
\begin{aligned}
F_{w}(x) & =\frac{\Gamma\left(\frac{\alpha}{2}+1, \frac{\alpha+\lambda}{x^{2}}\right)}{\Gamma\left(\frac{\alpha}{2}+1\right)} \\
& =1-\frac{\gamma\left(\frac{\alpha}{2}+1, \frac{\alpha+\lambda}{x^{2}}\right)}{\Gamma\left(\frac{\alpha}{2}+1\right)} .
\end{aligned}
$$

Here $\Gamma\left(\frac{\alpha}{2}+1, \frac{\alpha+\lambda}{x^{2}}\right)$ is an upper incomplete Gamma function defined by

$$
\begin{aligned}
\Gamma(a, x) & =\int_{x}^{\infty} t^{a-1} e^{-t} d t . \\
\Gamma(a, x) & =\Gamma(a)-\gamma(a, x),
\end{aligned}
$$

where $\gamma(a, x)$ is a lower incomplete Gamma function as

$$
\gamma(a, x)=\int_{0}^{x} t^{a-1} e^{-t} d t .
$$

In FIG. 2.1, different $p d f$ and $c d f$ plots of the $N W I R$ distribution are presented for the selected values of parameters $\alpha$ and $\lambda$. Now, let $Y=(\alpha+\lambda) X^{-2}$, where $X$ has the NWIR distribution with parameters $\alpha$ and $\lambda$. The $p d f$ of the random variable $Y$ becomes

$$
f(y)=\frac{1}{\Gamma\left(\frac{\alpha}{2}+1\right)} y^{\frac{\alpha}{2}} e^{-y}
$$

for $y>0$. Thus, the random variable $Y$ has a Gamma distribution shown as $Y \sim \operatorname{Gamma}\left(\frac{\alpha}{2}+1,1\right)$. 

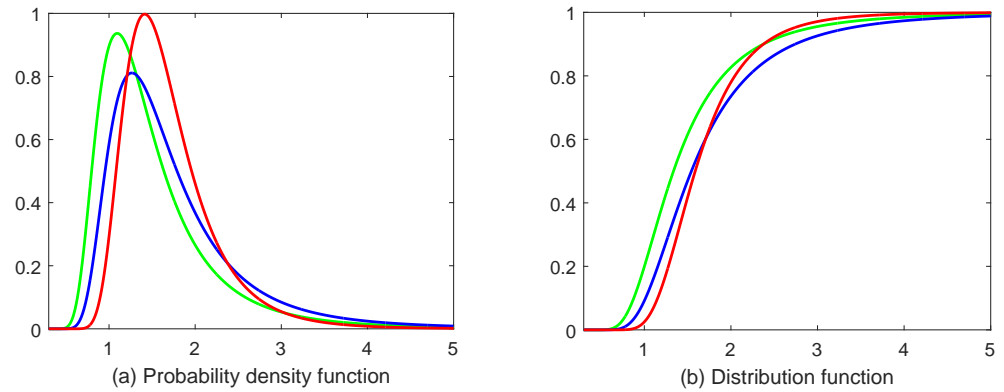

FIG. 2.1: Plots of the $p d f$ and $c d f$ of the $N W I R$ distribution where $\alpha=2, \lambda=1$ (green line); $\alpha=2, \lambda=2$ (blue line); $\alpha=5, \lambda=3$ (red line)

\section{Statistical and Reliability Properties}

In this section we consider some statistical and reliability properties of the NWIR distribution.

\section{1. $r^{\text {th }}$ moments}

If a random variable $X$ has the $N W I R$ distribution with a scale parameter $\lambda$ and shape parameter $\alpha$, then the $r^{t h}$ moment of the $N W I R$ distributed random variable $X$ is obtained as

$$
E\left(X^{r}\right)=\int_{0}^{\infty} \frac{2(\alpha+\lambda)^{\frac{\alpha}{2}+1}}{\Gamma\left(\frac{\alpha}{2}+1\right)} x^{r-\alpha-3} e^{-(\alpha+\lambda) x^{-2}} d x
$$

In order to calculate $E\left(X^{r}\right)$, using the transformation in (2.3), we obtain

$$
E\left(X^{r}\right)=(\alpha+\lambda)^{\frac{r}{2}} \frac{\Gamma\left(\frac{\alpha-r}{2}+1\right)}{\Gamma\left(\frac{\alpha}{2}+1\right)} .
$$

Hence, from the $r^{\text {th }}$ moment of the NWIR distribution, the first four moments can be easily calculated to obtain the mean, variance, coefficient of skewness and the coefficient of kurtosis of the NWIR distribution as follows

$$
\begin{aligned}
E(X) & =(\alpha+\lambda)^{\frac{1}{2}} \frac{\Gamma\left(\frac{\alpha+1}{2}\right)}{\Gamma\left(\frac{\alpha}{2}+1\right)}, \\
E\left(X^{2}\right) & =\frac{2(\alpha+\lambda)}{\alpha} \\
E\left(X^{3}\right) & =(\alpha+\lambda)^{\frac{3}{2}} \frac{\Gamma\left(\frac{\alpha-3}{2}+1\right)}{\Gamma\left(\frac{\alpha}{2}+1\right)}
\end{aligned}
$$


and

$$
E\left(X^{4}\right)=(\alpha+\lambda)^{2} \frac{\Gamma\left(\frac{\alpha-4}{2}+1\right)}{\Gamma\left(\frac{\alpha}{2}+1\right)} .
$$

\subsection{Moment generating function}

The moment generating function of the $N W I R$ distribution is given as follows. formula

$$
\begin{aligned}
M_{X}(t) & =E\left(e^{t x}\right) \\
& =\int_{0}^{\infty} e^{t x} \frac{2(\alpha+\lambda)^{\frac{\alpha}{2}+1}}{\Gamma\left(\frac{\alpha}{2}+1\right)} x^{-(\alpha+3)} e^{-(\alpha+\lambda) x^{-2}} d x .
\end{aligned}
$$

By applying the Maclaurin series $e^{t x}=\sum_{n=0}^{\infty} \frac{(t x)^{n}}{n !}$ and setting the transformation in (2.3), we finally get

$$
M_{X}(t)=\frac{1}{\Gamma\left(\frac{\alpha}{2}+1\right)} \sum_{n=0}^{\infty} \frac{t^{n}}{n !}(\alpha+\lambda)^{\frac{n}{2}} \Gamma\left(\frac{\alpha-n}{2}+1\right) .
$$

\subsection{Quantile function}

The quantile function of the NWIR distribution is obtained by

$$
x_{q}=F_{w}^{-1}(q), 0<q<1,
$$

where $F_{w}^{-1}(q)$ is the inverse of $c d f$ in (2.4). The median of the NWIR distributed random variable $X$ can be found by putting $q=0.5$ in (3.1). $F_{w}^{-1}(q)$ can be computed numerically via some mathematical and statistical software packages since it does not have a closed-form expression. Moreover, the equation in (3.1) can be used in order to generate a random number from the proposed distribution.

\subsection{Mode}

Now, the natural logarithm of the $f_{w}(x)$ in $(2.2)$ is given by

$$
\ln f_{w}(x) \propto-(\alpha+3) \ln x-(\alpha+\lambda) x^{-2} .
$$

Using the differentiating equation (3.2) with respect to $x$, we obtain as

$$
\frac{d}{d x} \ln f_{w}(x)=-(\alpha+3) x^{-1}+2(\alpha+\lambda) x^{-3} .
$$


If the equation (3.3) is equal to 0 and solve for $x$, then the mode of the NWIR distribution has the following expression

$$
X_{M}=\sqrt{\frac{2(\alpha+\lambda)}{\alpha+3}}
$$

for $\alpha>0$ and $\lambda>0$. Note that $f_{w}(x)$ is increasing when $x \in\left(0, X_{M}\right)$ and is decreasing when $x \in\left(X_{M}, \infty\right)$.

\subsection{Shannon entropy}

The statistical entropy introduced by Shannon [22] is defined as a measure of the information content associated with the outcome of a random variable (see [2]). The Shannon entropy of the NWIR distribution is expressed by

$$
\begin{aligned}
I_{S}(\alpha, \lambda)= & -E\left(\ln f_{w}(x)\right) \\
= & \ln \left(\frac{\Gamma\left(\frac{\alpha}{2}+1\right)}{2(\alpha+\lambda)^{\frac{\alpha}{2}+1}}\right)+(\alpha+3) E(\ln x) \\
& +(\alpha+\lambda) E\left(x^{-2}\right) .
\end{aligned}
$$

To calculate $E(\ln x)$, if we use the transformation in $(2.3)$, then we have

$$
\begin{aligned}
E(\ln x) & =\frac{1}{2 \Gamma\left(\frac{\alpha}{2}+1\right)} \int_{0}^{\infty} u^{\frac{\alpha}{2}}(\ln (\alpha+\lambda)-\ln u) e^{-u} d u \\
& =\frac{1}{2}\left(\ln (\alpha+\lambda)-\Psi\left(\frac{\alpha}{2}+1\right)\right),
\end{aligned}
$$

where $\Psi$ is a digamma function with

$$
\Psi(r)=\frac{d}{d r} \ln \Gamma(r)=\frac{\Gamma^{\prime}(r)}{\Gamma(r)}, r>0
$$

defined as the logarithmic derivative of the Gamma function. It is also well known that the derivative of $\Gamma(r)$ is

$$
\Gamma^{\prime}(r)=\int_{0}^{\infty} t^{r-1}(\ln t) e^{-t} d t .
$$

Substituting $E\left(x^{-2}\right)=\frac{\frac{\alpha}{2}+1}{\alpha+\lambda}$ and (3.5) into (3.4), Shannon entropy of the $N W I R$ distribution $I_{S}(\alpha, \lambda)$ becomes

$$
\begin{aligned}
I_{S}(\alpha, \lambda)= & \ln \left(\frac{\Gamma\left(\frac{\alpha}{2}+1\right)}{2(\alpha+\lambda)^{\frac{\alpha}{2}+1}}\right)+\left(\frac{\alpha}{2}+1\right) \\
& +\frac{(\alpha-3)}{2}\left(\ln (\alpha+\lambda)-\Psi\left(\frac{\alpha}{2}+1\right)\right) .
\end{aligned}
$$




\subsection{Rényi entropy}

Rényi entropy considered by Rényi [18] is a generalization of the Shannon entropy. The Rényi entropy of the NWIR distribution is expressed by

$$
\begin{aligned}
I_{R}(\delta) & =\frac{1}{1-\delta} \ln \int_{0}^{\infty} f_{w}^{\delta}(x) d x \\
& =\frac{1}{1-\delta} \ln \int_{0}^{\infty}\left(\frac{2(\alpha+\lambda)^{\frac{\alpha}{2}+1}}{\Gamma\left(\frac{\alpha}{2}+1\right)} x^{-(\alpha+3)} e^{-(\alpha+\lambda) x^{-2}}\right)^{\delta} d x \\
& =\frac{1}{1-\delta}\left(\delta \ln \frac{2(\alpha+\lambda)^{\frac{\alpha}{2}+1}}{\Gamma\left(\frac{\alpha}{2}+1\right)}+\ln \int_{0}^{\infty} x^{-\delta(\alpha+3)} e^{-\delta(\alpha+\lambda) x^{-2}} d x\right),
\end{aligned}
$$

where $\delta \neq 1$ and $\delta>0$. By using the transformation in (2.3), we obtain that

$$
\begin{aligned}
I_{R}(\delta)= & \frac{1}{1-\delta}\left(\ln 2^{\delta-1}+\left(\frac{1-\delta}{2}\right) \ln (\alpha+\lambda)-\delta \ln \Gamma\left(\frac{\alpha}{2}+1\right)\right) \\
& +\frac{1}{1-\delta}\left(\ln \Gamma\left(\frac{\delta(\alpha+3)-1}{2}\right)-\frac{\delta(\alpha+3)-1}{2} \ln \delta\right) .
\end{aligned}
$$

\subsection{Survival and hazard rate functions}

The survival and hazard rate functions of the NWIR distribution are defined by

$$
\begin{aligned}
S(x) & =1-F_{w}(x) \\
& =\frac{\gamma\left(\frac{\alpha}{2}+1, \frac{\alpha+\lambda}{x^{2}}\right)}{\Gamma\left(\frac{\alpha}{2}+1\right)},
\end{aligned}
$$

and

$$
\begin{aligned}
H(x) & =\frac{f_{w}(x)}{S(x)} \\
& =\frac{2(\alpha+\lambda)^{\frac{\alpha}{2}+1}}{\gamma\left(\frac{\alpha}{2}+1, \frac{\alpha+\lambda}{x^{2}}\right)} x^{-(\alpha+3)} e^{-(\alpha+\lambda) x^{-2}}
\end{aligned}
$$

for $x>0$, respectively. In FIG. 3.1, the graphs of the survival and hazard rate functions, which are plotted against different values of the parameters $\alpha$ and $\lambda$, are demonstrated.

Then, to determine the behavior of the hazard rate function of the NWIR distribution, the lemma established by Glaser [5] is used. Now, we define

$$
\begin{aligned}
\eta(x) & =-\frac{f_{w}^{\prime}(x)}{f_{w}(x)} \\
& =(\alpha+3) x^{-1}-2(\alpha+\lambda) x^{-3},
\end{aligned}
$$


and

$$
\eta^{\prime}(x)=-(\alpha+3) x^{-2}+6(\alpha+\lambda) x^{-4},
$$

where $f_{w}^{\prime}(x)$ is derivative of $p d f$ of the $N W I R$ distribution with respect to $x$. Thus, $\eta^{\prime}(x)=0$ provides when $x_{0}=\sqrt{\frac{6(\alpha+\lambda)}{\alpha+3}}$ for $\lambda>0, \alpha>0$. Note that, $\eta^{\prime}(x)>0$ and $\eta^{\prime}\left(x_{0}\right)=0$ when $0<x<x_{0}$ and $\eta^{\prime}(x)<0$ when $x>x_{0}$. Therefore, the hazard rate function of the $N W I R$ distribution is an upside down bathtub shape (see [19] and $[23])$.
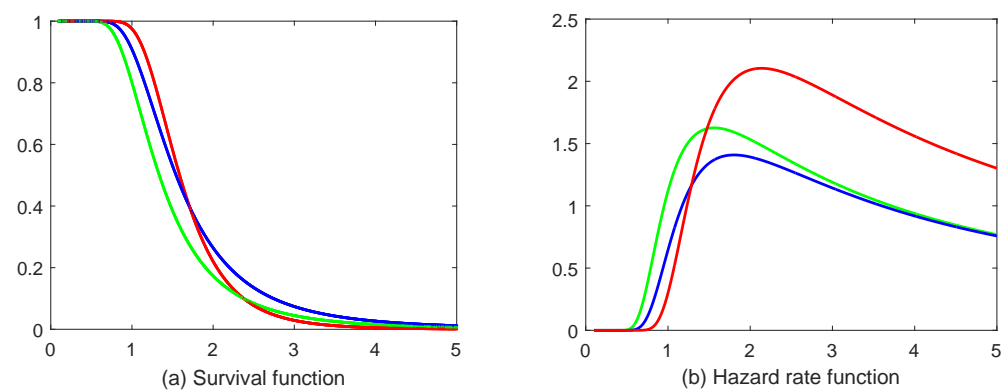

FIG. 3.1: Plots of the survival and hazard rate functions of the NWIR distribution where $\alpha=2, \lambda=1$ (green line); $\alpha=2, \lambda=2$ (blue line); $\alpha=5, \lambda=3$ (red line)

\subsection{Order statistics}

Let $X_{(1)}, X_{(2)}, \ldots, X_{(n)}$ be order statistics of a random sample $X_{1}, X_{2}, \ldots, X_{n}$ from the NWIR distribution. It is well known that the $p d f$ of $r^{\text {th }}$ order statistic $X_{(r)}(r=1,2, \ldots, n)$ is given as:

$$
f_{r: n}(x ; \alpha, \lambda)=r\left(\begin{array}{l}
n \\
r
\end{array}\right) f(x)(F(x))^{r-1}(1-F(x))^{n-r} .
$$

Applying the binomial series expansion of $(1-F(x))^{n-r}$ in (3.6), we get

$$
f_{r: n}(x ; \alpha, \lambda)=\sum_{k=0}^{n-r} r\left(\begin{array}{c}
n \\
r
\end{array}\right)\left(\begin{array}{c}
n-r \\
k
\end{array}\right)(-1)^{k} f(x)(F(x))^{r+k-1}
$$

After substituting (2.2) and (2.4) into (3.7), if we put the binomial series expansion of $(F(x))^{r+k-1}$ in $(3.7)$, then we have 


$$
\begin{aligned}
f_{r: n}(x ; \alpha, \lambda)= & \sum_{k=0}^{n-r} \sum_{t=0}^{r+k-1} 2(-1)^{r+2 k-1} \\
& \left.\times\left[\begin{array}{c}
r \\
r
\end{array}\right)\left(\begin{array}{c}
n-r \\
k
\end{array}\right)\left(\begin{array}{c}
r+k-1 \\
t
\end{array}\right)\right] \\
& \times\left[\frac{(\alpha+\lambda)^{\frac{\alpha}{2}+1} \gamma^{r+k-1}\left(\frac{\alpha}{2}+1, \frac{\alpha+\lambda}{x^{2}}\right)}{\Gamma^{r+k}\left(\frac{\alpha}{2}+1\right)}\right] \\
& \times\left[x^{-(\alpha+3)} e^{-(\alpha+\lambda) x^{-2}}\right] .
\end{aligned}
$$

Thus, the $p d f \mathrm{~s}$ of the smallest order statistic $X_{(1)}$ and largest order statistic $X_{(n)}$ can be obtained by writing the $r=1$ and $r=n$ in (3.8), respectively.

\section{Estimation}

Let $\left\{X_{1}, X_{2}, \ldots, X_{n}\right\}$ be a random sample from the NWIR distribution. The log-likelihood function of the sample is

$$
\begin{aligned}
\ln L(\alpha, \lambda \mid \underline{x})= & n \ln 2+n\left(\frac{\alpha}{2}+1\right) \ln (\alpha+\lambda)-n \ln \Gamma\left(\frac{\alpha}{2}+1\right) \\
& -(\alpha+3) \sum_{i=1}^{n} \ln x_{i}-(\alpha+\lambda) \sum_{i=1}^{n} x_{i}^{-2} .
\end{aligned}
$$

By differentiating (4.1) with respect to parameters $\alpha$ and $\lambda$, we have normal equations as

$$
\begin{aligned}
\frac{\partial \ln L(\alpha, \lambda \mid \underline{x})}{\partial \alpha}= & \frac{n}{2} \ln (\alpha+\lambda)+n \frac{\left(\frac{\alpha}{2}+1\right)}{\alpha+\lambda}-\frac{n}{2} \Psi\left(\frac{\alpha}{2}+1\right) \\
& -\sum_{i=1}^{n} \ln x_{i}-\sum_{i=1}^{n} x_{i}^{-2}=0 \\
\frac{\partial \ln L(\alpha, \lambda \mid \underline{x})}{\partial \lambda}= & n \frac{\left(\frac{\alpha}{2}+1\right)}{\alpha+\lambda}-\sum_{i=1}^{n} x_{i}^{-2}=0,
\end{aligned}
$$

where $\Psi\left(\frac{\alpha}{2}+1\right)=\frac{d}{d \alpha} \ln \Gamma\left(\frac{\alpha}{2}+1\right)=\frac{\Gamma^{\prime}\left(\frac{\alpha}{2}+1\right)}{\Gamma\left(\frac{\alpha}{2}+1\right)}$. Note that the solution of the equations in (4.2)-(4.3) gives maximum likelihood estimators $\widehat{\alpha}$ and $\hat{\lambda}$ of parameters $\alpha$ and $\lambda$. However, they do not have a closed form solution, and we must use numerical methods to solve them. Now, to give asymptotically a lower bound for the covariance matrix of $\widehat{\alpha}$ and $\widehat{\lambda}$, the Fisher information matrix is provided as a minus expected value of the second-order partial derivatives of the log-likelihood function 
under the regularity conditions, see [11]. It is defined by

$$
I_{n}(\alpha, \lambda)=\left[\begin{array}{cc}
-E\left(\frac{\partial^{2} \ln L(\alpha, \lambda \mid \underline{x})}{\partial \alpha^{2}}\right) & -E\left(\frac{\partial^{2} \ln L(\alpha, \lambda \mid \underline{x})}{\partial \alpha \partial \lambda}\right) \\
-E\left(\frac{\partial^{2} \ln L(\alpha, \lambda \mid \underline{x})}{\partial \lambda \partial \alpha}\right) & -E\left(\frac{\partial^{2} \ln L(\alpha, \lambda \mid \underline{x})}{\partial \lambda^{2}}\right)
\end{array}\right],
$$

and the elements of the matrix are obtained as follows

$$
\begin{aligned}
& E\left(\frac{\partial^{2} \ln L(\alpha, \lambda \mid \underline{x})}{\partial \alpha^{2}}\right)=\frac{n}{(\alpha+\lambda)}-n \frac{\left(\frac{\lambda}{2}+1\right)}{(\alpha+\lambda)^{2}}-\frac{n}{4} \Psi^{\prime}\left(\frac{\alpha}{2}+1\right) \\
& E\left(\frac{\partial^{2} \ln L(\alpha, \lambda \mid \underline{x})}{\partial \lambda^{2}}\right)=-n \frac{\left(\frac{\alpha}{2}+1\right)}{(\alpha+\lambda)^{2}} \\
& E\left(\frac{\partial^{2} \ln L(\alpha, \lambda \mid \underline{x})}{\partial \alpha \partial \lambda}\right)=n \frac{\left(\frac{\lambda}{2}-1\right)}{(\alpha+\lambda)^{2}}
\end{aligned}
$$

where $\Psi^{\prime}\left(\frac{\alpha}{2}+1\right)$ is first derivative of $\Psi\left(\frac{\alpha}{2}+1\right)$ with respect to $\alpha$. Therefore, maximum likelihood estimators of parameters $\alpha$ and $\lambda$ have asymptotically normal distribution with mean vector $\underline{0}$ and the covariance matrix $I_{n}^{-1}(\alpha, \lambda)$ as

$$
\sqrt{n}(\widehat{\alpha}-\alpha, \widehat{\lambda}-\lambda) \rightarrow N_{2}\left(\underline{0}, I_{n}^{-1}(\alpha, \lambda)\right)
$$

where $I_{n}^{-1}(\alpha, \lambda)$ is inverse of $I_{n}(\alpha, \lambda)$.

\section{An Application}

In this section, we consider a real data set, which is the daily mean wind speed data for March, taken in 2015 from the Turkish Meteorological Services for Sinop, Turkey, to demonstrate the practicability of the proposed distribution over the $I R$ and WIR (proposed by Fatima and Ahmad [8]) distributions, see Table 5.1.

Table 5.1: The daily mean wind speed data

\begin{tabular}{|l|l|l|l|l|l|l|l|}
\hline 2.8 & 1.8 & 3.2 & 5.0 & 2.4 & 4.8 & 2.9 & 2.9 \\
2.3 & 3.2 & 2.3 & 2.0 & 1.9 & 3.3 & 4.4 & 6.7 \\
4.3 & 1.9 & 2.2 & 3.3 & 2.1 & 4.0 & 2.0 & 3.1 \\
3.8 & 3.1 & 3.2 & 3.4 & 2.8 & 2.1 & 3.1 & \\
\hline
\end{tabular}

The Kolmogorov-Smirnov $(K-S)$ test, which is the one of the widely used goodness of fit tests, has been applied to verify that distributions fit to the real data set. The results of the $K-S$ test indicate that the $N W I R, W I R$ and $I R$ distributions are suitable for modeling the data set since the computed $K-S$ test values are less than theoretical $K-S$ test value $\left(K-S_{0.05 ; 31}=0.24\right)$, see Table 5.2 . 
Then, we determined which distribution better fits the real data set using model evaluating tests, i.e., the root mean square error (RMSE), the coefficient of determination $\left(R^{2}\right)$, ln-likelihood $(\ln L)$ and the Akaike information criterion $(A I C)$.

The tests results demonstrate that the $N W I R$ distribution gives a better fit to the data set compared to the WIR and $I R$ distributions because it has minimum $R M S E$ and $A I C$ and maximum $R^{2}$ and $\ln L$ values among the other distributions (see Table 5.2 and FIG. 5.1). Additionally, it was observed that there is no difference between the fitting performances of the $W I R$ and $I R$ distributions for the wind speed data (see FIG. 5.1).

Table 5.2: The $M L$ estimates of parameters and results of the $K-S$ test, $R M S E, R^{2}$, $\ln L$ and $A I C$ for the wind speed data

\begin{tabular}{|l|c|r|c|c|c|c|r|}
\hline Distribution & $\hat{\alpha}$ & \multicolumn{1}{|c|}{$\hat{\lambda}$} & $K-S$ & $R M S E$ & $R^{2}$ & $\ln L$ & \multicolumn{1}{c|}{$A I C$} \\
\hline NWIR & 3.7934 & 17.1586 & 0.0971 & 0.0532 & 0.9687 & -41.2814 & 86.5629 \\
WIR & 0.0100 & 7.1969 & 0.2398 & 0.1162 & 0.6691 & -48.7263 & 101.4525 \\
$I R$ & - & 7.2331 & 0.2393 & 0.1158 & 0.6729 & -48.6648 & 101.3290 \\
\hline
\end{tabular}

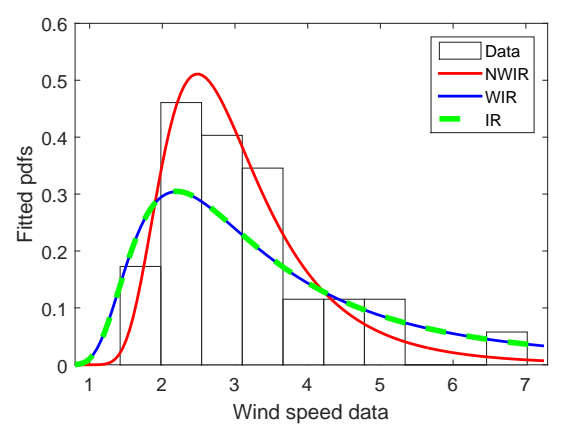

FiG. 5.1: Fitted plots and histogram for the data

\section{Conclusion}

In this study, a new weighted $I R$ distribution based on two different weight functions has been introduced. Moments, the moment generating function, survival and hazard rate functions, order statistics and entropy measures of the new distribution have been derived. The estimating equations have been provided in order to obtain $M L$ estimates of the individual parameters, and the Fisher information matrix has been derived in order to obtain approximate confidence intervals of the parameters. The relationship between the NWIR distribution and the Gamma distribution has also been proved. 
The applicability and superiority of the proposed distribution over the WIR and $I R$ distributions have been illustrated with real data. Therefore, the NWIR distribution can be considered as an alternative model for the statistical data analysis in wind speed studies and other fields.

\section{REF EREN C ES}

1. A. Ahmad , S. P. Ahmad and A. Ahmed: Transmuted inverse Rayleigh distribution: a generalization of the inverse Rayleigh distribution. Mathematical Theory and Modeling 4(7) (2014), 90-98.

2. S. F. Bush: Nanoscale Communication Networks. Artech House, 2010.

3. R. A. FISHER: The effects of methods of ascertainment upon the estimation of frequencies. Annals of Eugenics 6 (1934), 13-25.

4. M. E. Ghitany, F. Alqallaf , D. K. Al-Mutairi and H. A. Husain: A twoparameter weighted Lindley distribution and its applications to survival data. Mathematics and computers in Simulation 81(6) (2011), 1190-1201.

5. R. E. GLASER: Bathtub and related failure rate characterizations. Journal of American Statistical Association 75 (1980), 667-672.

6. R. C. Gupta and J. P. KeAting: Relations for reliability measures under length biased sampling. Scandinavian Journal of Statistics 13 (1986), 49-56.

7. R. C. Gupta and S. N. KIRMAni: The role of weighted distributions in stochastic modeling. Communications in Statistics-Theory and methods 19(9) (1990), 3147-3162.

8. K. FAtima and S. P. Ahmad: Weighted inverse Rayleigh distribution. International Journal of Statistics and Systems 12(1) (2017), 119-137.

9. J. X. KerseY: Weighted inverse Weibull and beta-inverse Weibull distribution. Georgia Southern University, 2010.

10. M. S. Khan: Modified inverse Rayleigh distribution. International Journal of Computer Applications 87(13) (2014), 28-33.

11. A. Klein and G. MÉLARD: Computation of the Fisher information matrix for time series models. Journal of Computational and Applied Mathematics 64(1-2) (1995), $57-68$.

12. J. Leao, H. Saulo, M. Bourguignon, R. Cintra, L. Rgo and G. Cordeiro: On some properties of the beta inverse Rayleigh distribution. Chilean Journal of Statistics 4(2) (2013), 111-131.

13. V. Leiva, A. Sanhueza and J. M. Angulo: A length-biased version of the Birnbaum-Saunders distribution with application in water quality. Stochastic Environmental Research and Risk Assessment 23(3) (2009), 299-307.

14. B. O. Oluyede: On inequalities and selection of experiments for length biased distributions. Probability in the Engineering and Informational Sciences 13(2) (1999), 169-185.

15. A. K. NANDA and K. JAIN: Some weighted distribution results on univariate and bivariate cases. Journal of Statistical Planning and Inference 77(2) (1999), 169-180.

16. G. P. PATIL: Encountered data, statistical ecology, environmental statistics, and weighted distribution methods. Environmetrics 2(4) (1991), 377-423. 
17. C. R. RAO: On discrete distributions arising out of methods of ascertainment. The Indian Journal of Statistics, Series A, 27 (1965), 311-324.

18. A. RÉnYI: On measures of information and entropy. Statistics and Probability 1 (1961), 547-561.

19. R. Roman: Theoretical properties and estimation in weighted Weibull and related distributions. M. S. Thesis, Georgia Southern University, Georgia, 2010.

20. A. Saghir , S. Tazeem and I. Ahmad: The length-biased weighted exponentiated inverted Weibull distribution. Cogent Mathematics 3(1) (2016), 1267299.

21. M. Q. Shahbaz, S. Shahbaz and N. S. Butt: The Kumaraswamy inverse Weibull distribution. Pakistan Journal of Statistics and Operation Research 8(3) (2012), 479489.

22. E. Shannon: A mathematical theory of communication. The Bell System Technical Journal 27(10) (1948), 379-423.

23. V. Sherina and B. O. Oluyede: Weighted inverse Weibull distribution: statistical properties and applications. Theoretical Mathematics and Applications 4(2) (2014), $1-30$.

24. V. N. Trayer: Doklady Acad. Nauk, Belorus, 1964.

25. V. G. VodA: On the inverse Rayleigh random variable. Reports of Statistical Application Research 19 (1972), 13-21.

Demet Aydin

Sinop University

Department of Statistics

57000 Sinop, Turkey

daydin@sinop.edu.tr 